\title{
Perfil da sífilis gestacional e congênita em Nossa Senhora do Socorro, Sergipe
}

\author{
Profile of management and congenital syphilis in our Nossa Senhora do Socorro, Sergipe \\ Perfil de sífilis gestacional y congénita en Nossa Senhora do Socorro, Sergipe
}

Recebido: 20/01/2021 | Revisado: 27/01/2021 | Aceito: 02/02/2021 | Publicado: 08/02/2021

\author{
Ana Fatima Souza Melo de Andrade \\ ORCID: https://orcid.org/0000-0002-7024-6175 \\ Faculdade Estácio de Sá, Brasil \\ E-mail: anafatimamelo@hotmail.com \\ Verônica de Lourdes Sierpe Jeraldo \\ ORCID: https://orcid.org/0000-0001-9813-7969 \\ Universidade Tiradentes, Brasil \\ E-mail: veronica_sierpe@hotmail.com
}

\begin{abstract}
Resumo
Objetivou-se identificar a incidência anual e acumulada da sífilis gestacional e sífilis congênita. Tratou-se de um estudo de corte transversal com abordagem quantitativa em uma perspectiva crítica e reflexiva. Os dados foram obtidos a partir do Sistema de Informações de Agravos de Notificação e do Sistema de Informações sobre Nascidos Vivos, por meio da base de dados cedida pela Secretaria de Vigilância Epidemiológica e Informação da Secretaria Municipal de Saúde de Nossa Senhora do Socorro, no período de 2011 a 2015. A incidência anual média de sífilis gestacional e sífilis congênita no período estudado foi de 6,82 casos para cada 1.000 gestantes e de 18,52 casos por 1.000 nascidos vivos, respectivamente. Foi possível concluir que a transmissão vertical esteve relacionada às perdas de oportunidades diagnósticas e terapêuticas e mostram que ainda há um longo caminho a ser percorrido para que as taxas de sífilis congênita atinjam as preconizadas pela Organização Pan Americana da Saúde e pelo Ministério da Saúde.
\end{abstract}

Palavras-chave: Sífilis congênita; Saúde pública; Serviços de saúde.

\begin{abstract}
The objective was to identify the annual and accumulated incidence of gestational syphilis and congenital syphilis. It was a cross-sectional study with a quantitative approach in a critical and reflective perspective. Data were obtained from the Notifiable Diseases Information System and the Live Births Information System, through the database provided by the Epidemiological Surveillance and Information Department of the Municipal Health Department of Nossa Senhora do Socorro, during the period from 2011 to 2015. The average annual incidence of gestational syphilis and congenital syphilis in the studied period was 6.82 cases for every 1,000 pregnant women and 18.52 cases per 1,000 live births, respectively. It was possible to conclude that vertical transmission was related to the loss of diagnostic and therapeutic opportunities and show that there is still a long way to go before the rates of congenital syphilis reach those recommended by the Pan American Health Organization and the Ministry of Health.
\end{abstract}

Keywords: Syphilis congenital; Public health; Health services.

\section{Resumen}

El objetivo fue identificar la incidencia anual y acumulada de sífilis gestacional y sífilis congénita. Fue un estudio transversal con un enfoque cuantitativo en una perspectiva crítica y reflexiva. Los datos se obtuvieron del Sistema de Información de Enfermedades Notificables y del Sistema de Información de Nacidos Vivos, a través de la base de datos proporcionada por el Departamento de Información y Vigilancia Epidemiológica del Departamento Municipal de Salud de Nossa Senhora do Socorro, durante el período de 2011 a 2015. La incidencia media anual de sífilis gestacional y sífilis congénita en el período estudiado fue de 6,82 casos por cada 1.000 gestantes y 18,52 casos por cada 1.000 nacidos vivos, respectivamente. Se pudo concluir que la transmisión vertical estuvo relacionada con la pérdida de oportunidades diagnósticas y terapéuticas y mostrar que aún queda un largo camino por recorrer antes de que las tasas de sífilis congénita alcancen las recomendadas por la Organización Panamericana de la Salud y el Ministerio de Salud.

Palabras clave: Súfilis congénita; Salud pública; Servicios de salud.

\section{Introduçãa}

A sífilis é uma infecção sexualmente transmissível (IST) de caráter sistêmico causada pelo Treponema pallidum. A infecção é transmitida somente para humanos pelas vias sexual, vertical e transmissão por transfusão sanguínea, sendo 
importante o diagnóstico precoce, já que quando não diagnosticada precocemente pode gerar sequelas irreversíveis (Brasil, 2015). Os maiores índices de infecção detectados no país ocorrem predominantemente através do contato sexual (Brasil, 2015; WHO, 2016).

O diagnóstico tardio da sífilis gestacional (SG) predispõe o risco para sífilis congênita (SC), sendo a assistência do pré-natal inadequada, o principal responsável pelo aumento das altas taxas de sífilis congênita, o que deve alertar sobre a atuação do sistema de saúde vigente (Silva et al., 2010; Domingues et al., 2013).

A sífilis congênita é uma doença que pode ser prevenida, a depender de fatores como o período em que a gestante foi diagnosticada, a fase da patologia e o início do tratamento, sendo de extrema importância o tratamento concomitante do parceiro sexual (Domingues et al., 2013).

A Organização Pan Americana da Saúde (2010) estimou que na América Latina e Caribe, 330.000 gestantes soropositivas para sífilis não receberam o tratamento para a doença durante as consultas de pré-natal a cada ano, e que dois terços dos casos de sífilis em gestantes resultem em sífilis congênita. Em 2010, a cobertura do diagnóstico de gestantes sifilíticas que fizeram o pré-natal, nos países da região foi de apenas $61 \%$, sendo observadas coberturas de diagnóstico mais alto nos países do Caribe (82\%) e do Cone Sul (76\%), incluindo o Brasil (37\%) (OPAS, 2010).

Para gestantes com sífilis, em todo o território nacional, foi instituída a notificação compulsória, por meio da Portaria $n^{\circ} 33$ de 14 de julho de 2005. Observou-se no Brasil, na última década, um aumento de notificação de ocorrências de sífilis gestacional que pode ser reflexo, em parte, ao aprimoramento do sistema de vigilância epidemiológica e ao aumento da distribuição de testes rápidos. Nesta situação, a política de saúde denominada Rede Cegonha, instituída em 2011, contribuiu para a ampliação do acesso ao diagnóstico de sífilis em gestante no país (Brasil, 2016).

Os índices epidemiológicos para sífilis ainda permanecem elevados, tanto gestacionais como congênita, mundialmente. A reemergência da sífilis e o aumento do número de casos diagnosticados internacionalmente evidenciam a relevância do estudo, também sendo perceptível a existência de lacunas sobre pesquisas abordando o controle da alta incidência desse agravo de notificação compulsória, motivando assim a reflexão sobre o perfil atual da doença e suas implicações para a saúde da mulher (Newman et al., 2013).

Desta forma, objetivou-se identificar a incidência anual e acumulada da sífilis gestacional e congênita. Especificamente, caracterizar o perfil sociodemográfico e obstétrico das gestantes e puérperas sifilíticas, e determinar os fatores de risco associados à ocorrência de sífilis congênita no período entre 2011 a 2015 no município de Nossa Senhora do Socorro situado no Estado de Sergipe - Brasil.

\section{Metodologia}

Trata-se de um estudo de corte transversal, seccional com abordagem descritiva e quantitativa em uma perspectiva crítica e reflexiva. O estudo foi realizado no município de Nossa Senhora do Socorro, no estado de Sergipe. Os métodos utilizados no presente estudo foram baseados nas etapas que compõe a metodologia do estudo de Lima et al. (2013).

Os registros de casos de sífilis gestacional e congênita foram identificados através do Sistema de Informações de Agravos de Notificação (SINAN), por meio da base de dados cedida pela Secretaria de Vigilância Epidemiológica e Informação da Secretaria Municipal de Saúde de Nossa Senhora do Socorro. As informações de saúde foram disponibilizadas pelo programa de tabulação e tratamento dos dados: TabWin - DATASUS, implementado no município supracitado.

As fichas de notificação de sífilis gestacional e congênita de casos confirmados no período de 2011 a 2015 foram incluídas no estudo. Os casos não confirmados e registros repetidos foram excluídos das análises de dados. Os critérios de inclusão utilizados para definição de caso de sífilis gestacional foram: gestante que durante o pré-natal apresentasse evidência clínica e/ou sorologia não treponêmica (VDRL - Venereal Disease Research Laboratory) reagente, com teste treponêmico 
(teste rápido ou ELISA - Enzyme-Linked Immunosorbent Assay) positivo ou não realizado (Brasil, 2005). E para definir o caso de sífilis congênita foram considerados os seguintes critérios:

1) toda criança, aborto ou natimorto de mãe com evidência clínica ou sorológica para sífilis que não tenha sido tratada ou tenha recebido tratamento inadequado;

2) menores de 13 anos com evidências sorológicas para sífilis, desde que afastadas as possibilidades de manutenção de anticorpos maternos e de sífilis adquirida;

3) menores de 13 anos com sorologia não treponêmica reagente para sífilis e evidência clínica, radiológica ou liquórica para sífilis congênita; ou

4) evidência microbiológica da presença do Treponema pallidum na placenta, cordão umbilical ou tecido da criança, produto de aborto ou natimorto (Brasil, 2005).

A caracterização do perfil sociodemográfico e obstétrico das mulheres sifilíticas foi realizada por meio de um formulário específico, além da determinação da incidência da sífilis gestacional e congênita no período proposto. Os dados foram fornecidos pelo programa de tabulação e tratamento dos dados: TabWin - DATASUS, implementado no município do estudo.

Para análise estatística, os dados foram descritos por meio de frequências simples e percentuais e, para avaliar tendências nas proporções observadas ao longo dos anos foi utilizado o teste Qui-quadrado de tendência (associação linear por linear). O nível de significância adotado foi de 5\% e o software utilizado foi o R Core Team 2017®.

Foram estabelecidas as frequências absolutas e relativas de todos os casos de notificações compulsórias de sífilis gestacional e congênita do período de 2011 a 2015 no município de Nossa Senhora do Socorro do estado de Sergipe.

A incidência de sífilis gestacional e congênita foi calculada para cada ano do período de estudo utilizando dados populacionais do IBGE. As incidências foram expressas por 1.000 gestantes e 1.000 nascidos vivos respectivamente. A incidência acumulada gestacional foi calculada pelo número de casos de sífilis gestacional incidente no período de 2011 a 2015 sobre população de gestantes acumulada anualmente. Já na incidência cumulativa de sífilis congênita, o numerador foi representado pela incidência de casos de sífilis congênita no período de 2011 a 2015 sobre população de nascidos vivos acumulados anualmente. As incidências foram expressas por 1.000 gestantes e 1.000 nascidos vivos, respectivamente.

A fonte de dados utilizada sobre a população de gestantes foi fornecida pelo Sistema de Informações de Pré-Natal (SISPRENATAL), e a população de nascidos vivos foi fornecida pelo Sistema de Informações sobre Nascidos Vivos (SINASC) do município do estudo. O teste qui-quadrado de tendência foi utilizado para avaliar se houve uma tendência de crescimento da incidência da doença no período estudado. Um nível de significância de 5\% foi utilizado para definir um aumento estatisticamente significante. Os dados foram armazenados em planilhas utilizando os programas estatísticos Excel e Tabwin, e as análises foram realizadas utilizando os programas estatísticos Tabwin e R Core Team 2017.

Para investigar a presença de fatores de risco associados à ocorrência de sífilis congênita, foram comparados os casos de sífilis congênita com a população de nascidos vivos em Nossa Senhora do Socorro no mesmo período de estudo, utilizando as variáveis sobre características maternas e do pré-natal comuns aos bancos de dados do SINAN e SINASC, respectivamente.

Os dados foram descritos por meio de frequências simples e percentual quando categórica além de média e desvio padrão quando contínua. As características maternas e obstétricas das gestantes e puérperas foram comparadas por meio de frequências relativas e diferenças foram consideradas estatisticamente significantes quando o valor de $\mathrm{p}$ obtido pelo teste do qui-quadrado bicaudal foi $<0,05$. Foi utilizado o teste Qui-Quadrado de Pearson para verificar associação entre as variáveis categóricas e o teste $\mathrm{T}$ para verificar diferenças de média. O nível de significância adotado foi de 5\%. Os dados foram armazenados em planilhas utilizando os programas Excel e Tabwin, e o Software utilizado para análise estatística foi o R Core Team 2017. 
Foram observados os aspectos éticos que regem pesquisas com seres humanos, conforme Resolução 466/12 do Conselho Nacional de Saúde. O estudo foi aprovado pelo Comitê de Ética em Pesquisa da Universidade Tiradentes (UNIT), com número do parecer de aprovação no 1.905 .765 .

\section{Resultados}

Nas Figuras 1 e 2 é apresentada a incidência ascendente anual e acumulada, respectivamente, tanto da sífilis congênita quanto da sífilis em gestantes, revelando que em 2015 houve um aumento de aproximadamente 100\% para sífilis gestacional e de 75\% para sífilis congênita quando comparado ao ano de 2011. A sífilis congênita apresentou incidência média anual de 18,5 casos por mil nascidos vivos.

Figura 1 - Incidência (\%) anual de Sífilis Congênita entre nascidos vivos e Sífilis Gestacional no período 2011-2015, no município de Nossa Senhora do Socorro, Sergipe.

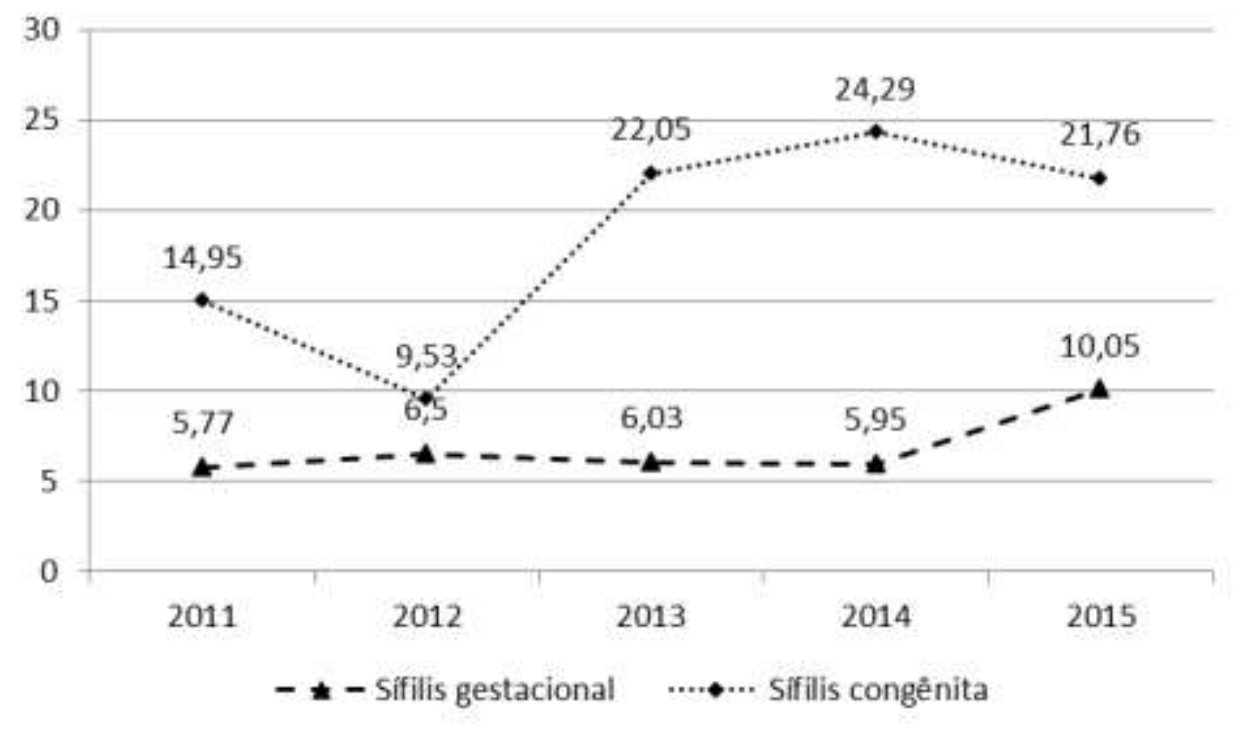

Fonte: Autores (2017).

Na Figura 1 é possível observar que a incidência anual de sífilis gestacional permaneceu constante de 2011 a 2014 , tendo uma elevação apenas no ano de 2015. A sífilis congênita apresentou tendência de ascendência todos os anos, exceto em 2012 (Figura 2). 
Figura 2 - Incidência (\%) acumulada de Sífilis Congênita entre nascidos vivos e Sífilis Gestacional no período 2011-2015 no município de Nossa Senhora do Socorro, Sergipe.

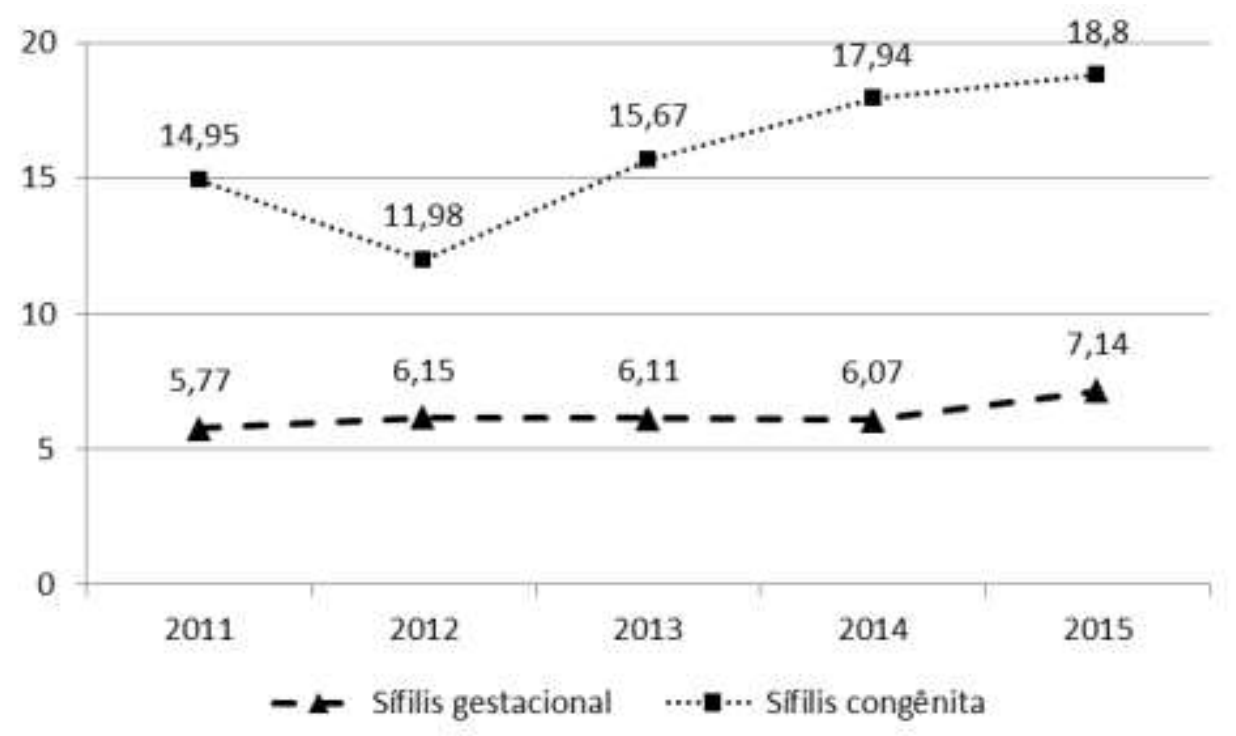

Fonte: Autores (2017).

A incidência acumulada geral foi de $2,06 \%$, sendo discrepante do valor normal dessa medida, que é de uma proporção de 0 a 1 (Figura 2).

Um total de 93 mulheres foram diagnosticadas com sífilis gestacional no período estudado. O perfil sóciodemográfico dessas mulheres, é apresentado na Tabela 1; revela que 69,9\% das mulheres eram de raça parda; e 78,5\% estavam na faixa etária de 14 a 30 anos, sendo que 38,7\% eram adolescentes entre 14 e 20 anos, sendo a idade da adolescência um fator considerado de risco para a sífilis gestacional. Com relação à escolaridade, 44,1\% possuíam o ensino fundamental incompleto, $19,4 \%$ ensino médio incompleto e $11,8 \%$ ensino médio completo.

Tabela 1 - Perfil sociodemográfico e obstétrico das gestantes/ mães portadoras de sífilis gestacional por ano de ocorrência de 2011-2015, Nossa Senhora do Socorro, Sergipe.

\begin{tabular}{llllllll}
\hline & 2011 & 2012 & 2013 & 2014 & 2015 & Total \\
& $\mathrm{N}(\%)$ & $\mathrm{N}(\%)$ & $\mathrm{N}(\%)$ & $\mathrm{N}(\%)$ & $\mathrm{N}(\%)$ & $\mathrm{N}(\%)$ & $\mathrm{p}$-valor \\
\hline $\begin{array}{l}\text { Perfil sociodemográfico } \\
\text { Raça }\end{array}$ & & & & & & & \\
\hline Ignorado & $3(23,1)$ & $1(6,3)$ & $0(0)$ & $2(13,3)$ & $0(0)$ & $6(6,5)$ & 0,650 \\
Branca & $2(15,4)$ & $1(6,3)$ & $3(21,4)$ & $2(13,3)$ & $5(14,3)$ & $13(14)$ & \\
Preta & $0(0)$ & $0(0)$ & $1(7,1)$ & $0(0)$ & $8(22,9)$ & $9(9,7)$ \\
Parda & $8(61,5)$ & $14(87,5)$ & $10(71,4)$ & $11(73,3)$ & $22(62,9)$ & $65(69,9)$ \\
Total & $\mathbf{1 3}(\mathbf{1 0 0})$ & $\mathbf{1 6}(\mathbf{1 0 0})$ & $\mathbf{1 4}(\mathbf{1 0 0})$ & $\mathbf{1 5}(\mathbf{1 0 0})$ & $\mathbf{3 5}(\mathbf{1 0 0})$ & $\mathbf{9 3}(\mathbf{1 0 0})$ \\
Faixa etária & & & & & & & \\
\hline 14 a 20 anos & $5(38,5)$ & $10(62,5)$ & $7(50)$ & $7(46,7)$ & $7(20)$ & $36(38,7)$ & 0,019 \\
21 a 30 anos & $5(38,5)$ & $5(31,3)$ & $5(35,7)$ & $6(40)$ & $16(45,7)$ & $37(39,8)$ \\
31 a 40 anos & $3(23,1)$ & $1(6,3)$ & $2(14,3)$ & $2(13,3)$ & $12(34,3)$ & $20(21,5)$ \\
Total & $\mathbf{1 3 ( 1 0 0 )}$ & $\mathbf{1 6}(\mathbf{1 0 0})$ & $\mathbf{1 4}(\mathbf{1 0 0})$ & $\mathbf{1 5}(\mathbf{1 0 0})$ & $\mathbf{3 5}(\mathbf{1 0 0})$ & $\mathbf{9 3}(\mathbf{1 0 0})$ \\
Escolaridade & & & & & & &
\end{tabular}




\begin{tabular}{llllllll}
\hline Ignorado & $4(30,8)$ & $1(6,3)$ & $2(14,3)$ & $4(26,7)$ & $4(11,4)$ & $15(16,1)$ & 0,758 \\
Analfabeto & $0(0)$ & $1(6,3)$ & $0(0)$ & $0(0)$ & $0(0)$ & $1(1,1)$ \\
$1^{\mathrm{a}} \mathrm{a} 4^{\mathrm{a}}$ série incompleta do EF & $1(7,7)$ & $3(18,8)$ & $2(14,3)$ & $0(0)$ & $8(22,9)$ & $14(15,1)$ \\
$4^{\mathrm{a}}$ série completa do EF & $0(0)$ & $2(12,5)$ & $0(0)$ & $1(6,7)$ & $4(11,4)$ & $7(7,5)$ \\
$5^{\mathrm{a}}$ a $8^{\mathrm{a}}$ série incompleta do EF & $1(7,7)$ & $6(37,5)$ & $3(21,4)$ & $4(26,7)$ & $6(17,1)$ & $20(21,5)$ \\
Ensino fundamental completo & $1(7,7)$ & $2(12,5)$ & $1(7,1)$ & $1(6,7)$ & $1(2,9)$ & $6(6,5)$ \\
Ensino médio incompleto & $4(30,8)$ & $0(0)$ & $3(21,4)$ & $4(26,7)$ & $7(20)$ & $18(19,4)$ \\
Ensino médio completo & $2(15,4)$ & $1(6,3)$ & $3(21,4)$ & $0(0)$ & $4(11,4)$ & $11(11,8)$ \\
Educação superior incompleta & $0(0)$ & $0(0)$ & $0(0)$ & $1(6,7)$ & $1(2,9)$ & $1(1,1)$ \\
Total & $\mathbf{1 3 ( 1 0 0 )}$ & $\mathbf{1 6}(\mathbf{1 0 0})$ & $\mathbf{1 4}(\mathbf{1 0 0})$ & $\mathbf{1 5}(\mathbf{1 0 0})$ & $\mathbf{3 5}(\mathbf{1 0 0})$ & $\mathbf{9 3}(\mathbf{1 0 0})$ \\
\hline
\end{tabular}

Perfil obstétrico

Teste Treponêmico

\begin{tabular}{lcllllll}
\hline Ignorado & $2(15,4)$ & $0(0)$ & $0(0)$ & $2(13,3)$ & $1(2,9)$ & $5(5,4)$ & 0,989 \\
Reativo & $7(53,8)$ & $10(62,5)$ & $11(78,6)$ & $11(73,3)$ & $22(62,9)$ & $61(65,6)$ & \\
Não reativo & $0(0)$ & $0(0)$ & $0(0)$ & $0(0)$ & $1(2,9)$ & $1(1,1)$ & \\
Não realizado & $4(30,8)$ & $6(37,5)$ & $3(21,4)$ & $2(13,3)$ & $11(31,4)$ & $26(28)$ & \\
$\begin{array}{l}\text { Total } \\
\text { Teste não treponêmico }\end{array}$ & $\mathbf{1 3}(\mathbf{1 0 0})$ & $\mathbf{1 6}(\mathbf{1 0 0})$ & $\mathbf{1 4}(\mathbf{1 0 0})$ & $\mathbf{1 5}(\mathbf{1 0 0})$ & $\mathbf{3 5}(\mathbf{1 0 0})$ & $\mathbf{9 3}(\mathbf{1 0 0})$ & \\
\hline Reativo & & & & & & & \\
Não reativo & $12(92,3)$ & $16(100)$ & $13(92,9)$ & $13(86,7)$ & $23(65,7)$ & $77(82,8)$ & 0,003 \\
Continuação de Tabela 1 & $0(0)$ & $0(0)$ & $0(0)$ & $0(0)$ & $1(2,9)$ & $1(1,1)$ & \\
Não realizado & $1(7,7)$ & $0(0)$ & $1(7,1)$ & $2(13,3)$ & $11(31,4)$ & $15(16,1)$ & \\
Total & $\mathbf{1 3 ( 1 0 0 )}$ & $\mathbf{1 6}(\mathbf{1 0 0})$ & $\mathbf{1 4}(\mathbf{1 0 0})$ & $\mathbf{1 5}(\mathbf{1 0 0})$ & $\mathbf{3 5}(\mathbf{1 0 0})$ & $\mathbf{9 3}(\mathbf{1 0 0})$ & \\
Classificação clínica da sífilis na gestante & & & & & & \\
\hline $\begin{array}{l}\text { Primária } \\
\text { Secundária }\end{array}$ & $4(30,8)$ & $0(0)$ & $1(7,1)$ & $1(6,7)$ & $4(11,4)$ & $10(10,8)$ & 0,347 \\
Terciária & $0(0)$ & $0(0)$ & $0(0)$ & $0(0)$ & $0(0)$ & $0(0)$ & \\
Latente & $0(0)$ & $0(0)$ & $0(0)$ & $0(0)$ & $0(0)$ & $0(0)$ & \\
Ignorado & $9(69,2)$ & $16(100)$ & $11(78,6)$ & $14(93,3)$ & $29(82,9)$ & $79(84,9)$ & \\
Total & $0(0)$ & $0(0)$ & $2(14,3)$ & $0(0)$ & $2(5,7)$ & $4(4,3)$ & \\
Esquema de tratamento da gestante & $\mathbf{1 3 ( 1 0 0 )}$ & $\mathbf{1 6}(\mathbf{1 0 0})$ & $\mathbf{1 4}(\mathbf{1 0 0})$ & $\mathbf{1 5}(\mathbf{1 0 0})$ & $\mathbf{3 5}(\mathbf{1 0 0})$ & $\mathbf{9 3}(\mathbf{1 0 0 )}$ & \\
\hline
\end{tabular}

\begin{tabular}{|c|c|c|c|c|c|c|c|c|c|}
\hline Ignorado & & & $1(7,7)$ & $0(0)$ & $0(0)$ & $0(0)$ & $0(0)$ & $1(1,1)$ & 0,006 \\
\hline $\begin{array}{l}\text { Penicilina } \\
2.400 .000 \mathrm{UI}\end{array}$ & G & benzantina & $2(15,4)$ & $0(0)$ & $0(0)$ & $0(0)$ & $0(0)$ & $2(2,2)$ & \\
\hline $\begin{array}{l}\text { Penicilina } \\
4.800 .000 \text { UI }\end{array}$ & G & benzantina & $1(7,7)$ & $0(0)$ & $0(0)$ & $0(0)$ & $0(0)$ & $1(1,1)$ & \\
\hline $\begin{array}{l}\text { Penicilina } \\
7.200 .000 \text { UI }\end{array}$ & G & benzantina & $8(61,5)$ & $16(100)$ & $14(100)$ & $14(93,3)$ & $34(97,1)$ & $86(92,5)$ & \\
\hline Outro esquema & & & $1(7,7)$ & $0(0)$ & $0(0)$ & $0(0)$ & $1(2,9)$ & $2(2,2)$ & \\
\hline Não realizado & & & $0(0)$ & $0(0)$ & $0(0)$ & $1(6,7)$ & $0(0)$ & $1(1,1)$ & \\
\hline Total & & & $13(100)$ & $16(100)$ & $14(100)$ & $15(100)$ & $35(100)$ & $93(100)$ & \\
\hline \multicolumn{10}{|c|}{ Parceiro tratado } \\
\hline Ignorado & & & $6(46,2)$ & $0(0)$ & $1(7,1)$ & $3(20)$ & $0(0)$ & $10(10,8)$ & 0,304 \\
\hline Sim & & & $5(38,5)$ & $6(37,5)$ & $5(35,7)$ & $8(53,3)$ & $24(68,6)$ & $48(51,6)$ & \\
\hline Não & & & $2(15,4)$ & $10(62,5)$ & $8(57,1)$ & $4(26,7)$ & $11(31,4)$ & $35(37,6)$ & \\
\hline Total & & & $13(100)$ & $16(100)$ & $14(100)$ & $15(100)$ & $35(100)$ & $93(100)$ & \\
\hline
\end{tabular}


Esquema de tratamento do parceiro

\begin{tabular}{|c|c|c|c|c|c|c|c|}
\hline Ignorado & $0(0)$ & $0(0)$ & $1(7,7)$ & $1(7,1)$ & $2(4,9)$ & $4(4,3)$ & 0,357 \\
\hline $\begin{array}{l}\text { Penicilina } \mathrm{G} \quad \text { benzantina } \\
2.400 .000 \mathrm{UI}\end{array}$ & $0(0)$ & $0(0)$ & $0(0)$ & $1(7,1)$ & $0(0)$ & $1(1,1)$ & \\
\hline $\begin{array}{l}\text { Penicilina } \quad G \\
4.800 .000 \mathrm{UI}\end{array}$ & $2(22,2)$ & $0(0)$ & $0(0)$ & $0(0)$ & $0(0)$ & $2(2,2)$ & \\
\hline $\begin{array}{l}\text { Penicilina G } \quad \text { benzantina } \\
7.200 .000 \text { UI }\end{array}$ & $4(44,4)$ & $7(43,8)$ & $5(38,5)$ & $8(57,1)$ & $24(58,5)$ & $48(51,6)$ & \\
\hline Outro esquema & $0(0)$ & $0(0)$ & $0(0)$ & $0(0)$ & $1(2,4)$ & $1(1,1)$ & \\
\hline Não realizado & $3(33,3)$ & $9(56,3)$ & $7(53,8)$ & $4(28,6)$ & $14(34,1)$ & $37(39,8)$ & \\
\hline Total & $9(100)$ & $16(100)$ & $13(100)$ & $14(100)$ & $41(100)$ & $93(100)$ & \\
\hline \multicolumn{8}{|c|}{ Motivo para o não tratamento do parceiro } \\
\hline Ignorado & $10(76,9)$ & $5(31,3)$ & $7(50)$ & $11(73,3)$ & $23(65,7)$ & $56(60,2)$ & 0,055 \\
\hline $\begin{array}{l}\text { Parceiro não teve mais contato } \\
\text { com a gestante }\end{array}$ & $0(0)$ & $1(6,3)$ & $1(7,1)$ & $1(6,7)$ & $4(11,4)$ & $7(7,5)$ & \\
\hline $\begin{array}{l}\text { Não convocado a UBS para } \\
\text { tratamento e não compareceu }\end{array}$ & $0(0)$ & $5(31,3)$ & $4(28,6)$ & $2(13,3)$ & $4(11,4)$ & $15(16,1)$ & \\
\hline $\begin{array}{l}\text { Convocado a UBS para } \\
\text { tratamento, mas recusou }\end{array}$ & $1(7,7)$ & $2(12,5)$ & $2(14,3)$ & $0(0)$ & $2(5,7)$ & $7(7,5)$ & \\
\hline Parceiro com sorologia reagente & $0(0)$ & $0(0)$ & $0(0)$ & $1(6,7)$ & $2(5,7)$ & $3(3,2)$ & \\
\hline Outro motivo & $2(15,4)$ & $3(18,8)$ & $0(0)$ & $0(0)$ & $0(0)$ & $5(5,4)$ & \\
\hline Total & $13(100)$ & $16(100)$ & $14(100)$ & $15(100)$ & $35(100)$ & $93(100)$ & \\
\hline
\end{tabular}

Fonte: Autores (2017).

Quanto ao perfil obstétrico das gestantes com sífilis gestacional outra variável que chama a atenção é a classificação clínica da sífilis e o tratamento realizado. A maioria (84,9\%) das gestantes foi classificada estando na fase de latência. Porém o tratamento foi realizado em 92,5\% dos casos com Penicilina benzatina 7.200.000 UI e os outros casos foram tratados seguindo diferentes esquemas de dosagem do mesmo medicamento ou mesmo outras medicações (Tabela 1).

Destaca-se que ao observar os percentuais de não tratamento do parceiro (37,6\%), tratamento do parceiro ignorado $(10,8 \%)$, tratamento da gestante não realizado $(1,1 \%)$, outro esquema de tratamento para gestante adotado $(2,2 \%)$ e tratamento da gestante ignorado $(1,1 \%)$, mais da metade $(52,8 \%)$ dos casos de sífilis em gestantes foram inadequadamente tratados. Dessas mulheres 81,4\% realizaram o pré-natal no município de Nossa Senhora do Socorro, Sergipe (Tabela 1).

Na tabela 1 também pode ser observado ao longo dos anos, que as variáveis faixa etária, teste não-treponêmico e esquema de tratamento da gestante, apresentaram um nível de significância menor que 5\%, isto é, apresentaram uma tendência de aumento ao longo do período estudado. Para o diagnóstico da sífilis gestacional, 83,9\% das gestantes realizaram o teste não treponêmico (VDRL) como triagem no pré-natal. Destas $82,8 \%$ foram reativos, porém ainda um percentual importante não realizou o exame (16\%). Para o teste treponêmico, $65,6 \%$ foi reativo, enquanto que $28 \%$ não foram realizados.

Com relação ao tratamento dos parceiros, apenas 51,6\% foram tratados com Penicilina G benzantina 7.200.000 UI, utilizado quando classificado como sífilis latente ou tardia. Já 37,6\% não foram tratados, destes 60,2\% a informação do motivo do não tratamento foi ignorado, $16,1 \%$ o parceiro foi convocado para UBS para tratamento, mas não compareceu, 7,5\% o parceiro não teve mais contato com a gestante (Tabela 1).

Um total de 269 crianças portadoras de sífilis foi registrado no Sistema Nacional de Agravos de Notificação (SINAN) no período estudado. A Tabela 2 mostra o perfil sociodemográfico e obstétrico das mães das crianças portadoras de sífilis congênita no período de 2011 a 2015 no município de Nossa Senhora de Socorro, bem como o tratamento adotado tanto para 
mãe quanto para o parceiro por meio da fonte de dados coletados das Fichas de Notificação de Sífilis Congênita. Não constando a variável raça, por falta de dados no instrumento de coleta.

Tabela 2 - Perfil sociodemográfico e obstétrico das mães das crianças portadoras de sífilis congênita por ano de ocorrência de 2011-2015, Nossa Senhora do Socorro, Sergipe.

\begin{tabular}{|c|c|c|c|c|c|c|c|}
\hline & $\begin{array}{l}2011 \\
\mathrm{~N}(\%)\end{array}$ & $\begin{array}{l}2012 \\
\mathrm{~N}(\%)\end{array}$ & $\begin{array}{l}2013 \\
\mathrm{~N}(\%)\end{array}$ & $\begin{array}{l}2014 \\
\mathrm{~N}(\%)\end{array}$ & $\begin{array}{l}2015 \\
\mathrm{~N}(\%)\end{array}$ & $\begin{array}{l}\text { Total } \\
\mathrm{N}(\%)\end{array}$ & $\mathrm{p}$-valor \\
\hline \multicolumn{8}{|c|}{$\begin{array}{l}\text { Perfil sociodemográfico } \\
\text { Faixa Etária }\end{array}$} \\
\hline 10 a 14 anos & $0(0)$ & $2(7,4)$ & $0(0)$ & $0(0)$ & $1(1,4)$ & $3(1,1)$ & 0,651 \\
\hline 15 a 19 anos & $8(22,2)$ & $6(22,2)$ & $20(30,3)$ & $16(22,5)$ & $12(17,4)$ & $62(23)$ & \\
\hline 20 a 34 anos & $23(63,9)$ & $14(51,9)$ & $38(57,6)$ & $45(63,4)$ & $46(66,7)$ & $\begin{array}{l}166 \\
(61,7)\end{array}$ & \\
\hline 35 a 49 anos & $4(11,1)$ & $5(18,5)$ & $7(10,6)$ & $10(14,1)$ & $10(14,5)$ & $36(13,4)$ & \\
\hline Ignorado & $1(2,8)$ & $0(0)$ & $1(1,5)$ & $0(0)$ & $1(1,4)$ & $2(0,7)$ & \\
\hline \multicolumn{8}{|l|}{$\begin{array}{l}\text { Perfil obstétrico } \\
\text { Realizou Pré Natal }\end{array}$} \\
\hline \multicolumn{8}{|l|}{ Continuação da Tabela 2} \\
\hline Sim & $22(62,9)$ & $17(63)$ & $47(71,2)$ & $41(57,7)$ & $55(78,6)$ & $\begin{array}{l}182 \\
(67,7)\end{array}$ & \\
\hline Não & $11(31,4)$ & $10(37)$ & $18(27,3)$ & $27(38)$ & $14(20)$ & $80(29,7)$ & \\
\hline Total & $35(100)$ & $27(100)$ & $66(100)$ & $71(100)$ & $70(100)$ & $269(100)$ & \\
\hline \multicolumn{8}{|c|}{ Momento do diagnóstico da sífilis materna } \\
\hline Ignorado & $0(0)$ & $0(0)$ & $1(1,5)$ & $1(1,4)$ & $1(1,4)$ & $3(1,1)$ & 0,508 \\
\hline Durante o pré-natal & $10(28,6)$ & $11(40,7)$ & $22(33,3)$ & $22(31)$ & $35(50)$ & $\begin{array}{l}100 \\
(37,2)\end{array}$ & \\
\hline $\begin{array}{l}\text { No momento do parto/ } \\
\text { curetagem }\end{array}$ & $19(54,3)$ & $16(59,3)$ & $36(54,5)$ & $38(53,5)$ & $20(28,6)$ & $129(48)$ & \\
\hline Após o parto & $6(17,1)$ & $0(0)$ & $7(10,6)$ & $10(14,1)$ & $13(18,6)$ & $36(13,4)$ & \\
\hline Não realizado & $0(0)$ & $0(0)$ & $0(0)$ & $0(0)$ & $1(1,4)$ & $1(0,4)$ & \\
\hline Total & $35(100)$ & $27(100)$ & $66(100)$ & $71(100)$ & $70(100)$ & $269(100)$ & \\
\hline \multicolumn{8}{|c|}{ Realização do teste treponêmico do parto } \\
\hline Ignorado & $0(0)$ & $0(0)$ & $1(1,5)$ & $0(0)$ & $1(1,4)$ & $2(0,7)$ & 0,450 \\
\hline Reagente & $35(100)$ & $27(100)$ & $65(98,5)$ & $71(100)$ & $67(95,7)$ & $\begin{array}{l}265 \\
(98,5)\end{array}$ & \\
\hline Não realizado & $0(0)$ & $0(0)$ & $0(0)$ & $0(0)$ & $2(2,9)$ & $2(0,7)$ & \\
\hline Total & $35(100)$ & $27(100)$ & $66(100)$ & $71(100)$ & 70 (100) & $269(100)$ & \\
\hline \multicolumn{8}{|c|}{ Realização do Teste Confirmatório Treponêmico no parto } \\
\hline Ignorado & $1(2,9)$ & $0(0)$ & $2(3)$ & $0(0)$ & $1(1,4)$ & $4(1,5)$ & $<0,001$ \\
\hline Reagente & $25(71,4)$ & $12(44,4)$ & $25(37,9)$ & $22(31)$ & $15(21,4)$ & $99(36,8)$ & \\
\hline Não reagente & $0(0)$ & $1(3,7)$ & $1(1,5)$ & $0(0)$ & $1(1,4)$ & $3(1,1)$ & \\
\hline Não realizado & $9(25,7)$ & $14(51,9)$ & $38(57,6)$ & $49(69)$ & $53(75,7)$ & $\begin{array}{l}163 \\
(60,6) \\
\end{array}$ & \\
\hline Total & 35 (100) & 27 (100) & $66(100)$ & $71(100)$ & 70 (100) & $269(100)$ & \\
\hline
\end{tabular}


Esquema de tratamento da mãe/ gestante

\begin{tabular}{llllllll}
\hline Ignorado & $1(2,9)$ & $0(0)$ & $1(1,5)$ & $0(0)$ & $1(1,4)$ & $3(1,1)$ & 0,013 \\
Adequado & $2(5,7)$ & $0(0)$ & $0(0)$ & $0(0)$ & $0(0)$ & $2(0,7)$ & \\
Inadequado & $15(42,9)$ & $25(92,6)$ & $59(89,4)$ & $65(91,5)$ & $61(87,1)$ & $\begin{array}{l}225 \\
(83,6)\end{array}$ & \\
Não realizado & $17(48,6)$ & $2(7,4)$ & $6(9,1)$ & $6(8,5)$ & $8(11,4)$ & $39(14,5)$ & \\
\hline Total & $\mathbf{3 5}(\mathbf{1 0 0})$ & $\mathbf{2 7}(\mathbf{1 0 0})$ & $\mathbf{6 6}(\mathbf{1 0 0})$ & $\mathbf{7 1 ( 1 0 0 )}$ & $\mathbf{7 0}(\mathbf{1 0 0})$ & $\mathbf{2 6 9}(\mathbf{1 0 0})$ & \\
Realização do tratamento do parceiro & & & & & \\
\hline Ignorado & $6(17,1)$ & $0(0)$ & $2(3)$ & $4(5,6)$ & $2(2,9)$ & $14(5,2)$ & 0,057 \\
Sim & $4(11,4)$ & $4(14,8)$ & $0(0)$ & $6(8,5)$ & $7(10)$ & $21(7,8)$ & \\
Não & $25(71,4)$ & $23(85,2)$ & $64(97)$ & $61(85,9)$ & $61(87,1)$ & $234(87)$ & \\
Total & $\mathbf{3 5 ( 1 0 0 )}$ & $\mathbf{2 7 ( 1 0 0 )}$ & $\mathbf{6 6}(\mathbf{1 0 0})$ & $\mathbf{7 1 ( 1 0 0 )}$ & $\mathbf{7 0}(\mathbf{1 0 0})$ & $\mathbf{2 6 9}(\mathbf{1 0 0})$ & \\
\hline
\end{tabular}

Fonte: Autores (2017).

As mães das crianças que apresentaram sífilis congênita em sua maioria estavam na faixa etária de 20 a 34 anos $(61,7 \%)$. Destas, $67,7 \%$ realizaram o pré-natal, porém apenas 37,2\% do diagnóstico foram realizados no período do pré-natal, $62,4 \%$ foi realizado no momento do parto/curetagem ou no pós-parto, isto quer dizer que o diagnóstico foi feito tardiamente. Com relação ao tratamento, $98,1 \%$ destes foram realizados de forma inadequada ou ignorados, e $87 \%$ dos parceiros não foram tratados (Tabela 2).

Em relação à realização dos testes para diagnóstico da sífilis congênita, observou-se que 98,5\% foram reagentes para o teste treponêmico do parto. $60,6 \%$ das gestantes não realizaram o teste confirmatório treponêmico no momento do parto e que das $37,9 \%$ que realizaram, 36,8\% foram reagentes. Quanto ao tratamento do parceiro, 92,2\% não realizaram tratamento (Tabela 2).

A Tabela 2 também expõe que ao avaliar as tendências nas proporções observadas ao longo dos anos as variáveis realização do teste treponêmico confirmatório no parto e esquema de tratamento da mãe apresentaram um nível de significância menor que 5\%, ou melhor, apresentaram uma tendência de aumento ao longo do período estudado.

A Tabela 3 demonstra que ao avaliar a associação entre as características sociodemográficas maternas e a ocorrência de sífilis congênita, pode-se observar que todas as variáveis: idade, escolaridade e estado civil da mãe apresentaram um nível de significância menor que 5\%, isto quer dizer que essas variáveis podem ser consideradas fatores de risco para a ocorrência da sífilis congênita (Tabela 3). 
Tabela 3 - Ocorrência de sífilis congênita de acordo com as características sociodemográficas e relativas ao pré-natal em Nossa Senhora do Socorro - SE, 2011 a 2015.

\begin{tabular}{|c|c|c|c|c|c|}
\hline $\begin{array}{l}\text { Características } \\
\text { sociodemográficas } \\
\text { relativas ao pré-natal }\end{array}$ & e $\mathbf{N}$ & $\begin{array}{l}\text { No. de casos } \\
\text { de sífilis } \\
\text { congênita }(\%)\end{array}$ & $\mathbf{N}$ & $\begin{array}{ll}\text { No. } & \text { de } \\
\text { nascidos } & \\
\text { vivos }(\%) & \end{array}$ & p-valor \\
\hline Estado Civil da Mãe & 206 & & 15484 & & \\
\hline Solteira & & $86(41,8)$ & & $4970(32,1)$ & 0,003 \\
\hline Casada & & $120(58,2)$ & & $10514(67,9)$ & \\
\hline Idade da Mãe & 208 & & 15548 & & \\
\hline$\leq 19$ & & $50(24,0)$ & & $3604(23,2)$ & $<0,001$ \\
\hline $20-34$ & & $130(62,5)$ & & $10444(67,2)$ & \\
\hline$\geq 35$ & & $28(13,5)$ & & $1500(9,6)$ & \\
\hline Raça/Cor da Mãe & 207 & & 15420 & & \\
\hline Branca & & $24(11,6)$ & & $1944(12,6)$ & $<0,001$ \\
\hline Preta & & $22(10,6)$ & & $1576(10,2)$ & \\
\hline Parda & & $161(77,8)$ & & $11900(77,2)$ & \\
\hline Escolaridade da Mãe & 208 & & 15502 & & \\
\hline Fundamental I & & $5(2,4)$ & & $106(0,7)$ & $<0,001$ \\
\hline Fundamental II & & $28(13,5)$ & & $822(5,4)$ & \\
\hline Médio & & $87(41,8)$ & & $4231(27,7)$ & \\
\hline Superior Incompleto & & $82(39,4)$ & & $9063(59,3)$ & \\
\hline Superior Completo & & $6(2,9)$ & & $1072(7,0)$ & \\
\hline
\end{tabular}

Nota: Teste Qui-Quadrado de Pearson. Fonte: Autores (2017).

\section{Discussão}

Neste estudo, a sífilis congênita apresentou incidência média anual de 18,5 casos por mil nascidos vivos, entretanto, esses valores estão longe da meta adotada pelo Ministério da Saúde para eliminação da SC, seguindo as orientações definidas pela Organização Mundial da Saúde (OMS) e a OPAS de 0,5 casos de SC para cada mil nascidos vivos (WHO, 2014; OPAS, 2014). O valor encontrado nesse estudo foi 37,04 vezes maior do que o valor definido como referência pela OMS em 2008.

Entre os estados do Brasil as taxas de notificações são semelhantes as encontradas neste estudo e revelam uma alta ocorrência de infecção pela sífilis, como em Belo Horizonte com um índice de 1,1 por mil nascidos vivos (Lima et al., 2013); em Campo Grande (MS), 23,4 casos por mil nascidos vivos (Figueiró et al., 2007); no Amazonas, 2,6 por mil nascidos vivos (Soeiro et al., 2014); em Sergipe, 11,2 casos por mil nascidos vivos para SC e 8,2 ocorrências por gestantes para a Sífilis Getacional (Brasil, 2015).

Os altos índices em Sergipe podem estar relacionados a diversos fatores, como possíveis falhas no tratamento da sífilis durante a gravidez, dependendo do estágio da doença e da idade gestacional, principalmente quando o diagnóstico é feito tardiamente, mesmo em mulheres que realizaram a quantidade mínima de consultas no pré-natal ou por falta de investigação epidemiológica de recém-nascidos (RNs) de gestantes positivas (Sheffield et al., 2002; Lafetá et al., 2016).

Foi possível observar que a incidência anual de sífilis gestacional permaneceu constante de 2011 a 2014, tendo uma elevação apenas no ano de 2015. Esse aumento pode estar relacionado a uma vigilância epidemiológica mais atuante, como também a ampliação do acesso ao diagnóstico de sífilis em gestantes no país (Brasil, 2016).

A sífilis congênita apresentou tendência de ascendência todos os anos, exceto em 2012. Acredita-se que a redução desse índice epidemiológico pode ser justificada pela atuação conjunta da Secretaria de Saúde e a Atenção Primária na adoção 
de medidas de controle da doença. Entretanto mostra também, que as medidas não foram tão eficazes durante os anos seguintes, podendo este fato estar relacionado à subnotificação da doença, ou erro de preenchimento das fichas de notificação já que os dados mostram que ocorrem falhas no registro e detecção da morbidade durante o período gravídico e no momento do nascimento (Soeiro et al., 2014; Campos et al., 2010; Brasil, 2015).

A incidência acumulada geral de Sífilis Congênita entre nascidos vivos e Sífilis Gestacional foi discrepante ao valor normal dessa medida, que é de uma proporção de 0 a 1 . O resultado encontrado justifica-se pela possível subnotificação de casos, resultante da disparidade de 93 casos notificados de sífilis gestacional para 269 casos notificados de sífilis congênita no período estudado. Como também pelo número de gestantes cadastradas no SISPRENATAL ser inferior ao número de nascidos vivos registrados no SINASC do município no mesmo período (Lafetá et al., 2016; Campos et al., 2010; Leitão et al., 2009).

Dessa forma, pode-se evidenciar que muitas gestantes do município de Nossa Senhora do Socorro não realizaram prénatal no município e área de saúde cadastrada ou não foram assistidas no período gravídico. A subnotificação também foi relatada em outras áreas brasileiras e revela uma falha importante na assistência à saúde materna e neonatal (Lafetá et al., 2016; Campos et al., 2010; Leitão et al., 2009).

No período investigado foi realizado o cálculo da incidência acumulada, para medir o risco de transmissão da sífilis de mãe para filho no recorte temporal relativo aos casos de gestantes notificadas para sífilis com ou sem a transmissão vertical. Os dados apontaram que a sífilis gestacional apresentou uma tendência constante, e que a sífilis congênita foi crescente nos anos em estudo, exceto no ano de 2012, sinalizando para uma elevada probabilidade de que a gestante com sífilis resultou em sífilis congênita por transmissão vertical no período, o que sugere falhas no seguimento pré-natal na área de estudo, uma vez que para redução das consequências da sífilis nos RNs, se faz necessário que as gestantes diagnosticadas com sífilis sejam adequadamente tratadas, durante a gestação, e em tempo oportuno (Gust et al., 2002).

Estudos afirmaram que a faixa etária da maioria das gestantes infectadas com sífilis está entre 20 a 29 anos. Corroborando com o perfil sociodemográfico das mulheres diagnosticadas com sífilis gestacional do presente estudo, que obteve dados semelhantes. Assim como, em um estudo transversal multicêntrico, envolvendo 24 estados brasileiros, com apoio do Programa Nacional IST/AIDS a gravidez na adolescência é um fator de risco para a ocorrência da sífilis gestacional (Domingues et al., 2013; Rodrigues et al., 2008).

Com relação à escolaridade, os resultados encontrados neste estudo condizem com uma característica mostrada também em outros estudos realizados no Brasil, como em Tocantins e em Manaus (Cavalcante et al., 2017; Soeiro et al., 2014). Sendo este um importante indicador no que diz respeito ao autocuidado. Outros autores admitem que, embora essa morbidade não seja uma doença restrita à classe menos favorecida, estabelece relações ao baixo nível socioeconômico e pode influenciar na restrição do acesso aos serviços de saúde (Figueiró et al., 2007; Mesquita et al., 2012).

Quanto ao perfil obstétrico das gestantes com sífilis gestacional outra variável que chama a atenção é a classificação clínica da sífilis e o tratamento realizado. A maioria das gestantes foi classificada estando na fase de latência, porém, a maioria do tratamento foi realizado com Penincilina benzatina 7.200.000 UI. Os outros casos foram tratados seguindo diferentes esquemas de dosagem do medicamento ou outras medicações. Estes dados mostram que o protocolo definido pelo Ministério da Saúde não foi cumprido adequadamente, ou na maioria das mulheres o diagnóstico foi feito tardiamente, demonstrando a fragilidade do serviço de saúde ou a baixa adesão das gestantes ao pré-natal (Brasil, \& Ministério da Saúde, 2006).

Na Turquia, observaram que quando a gestante positiva é adequadamente tratada, os bebês não apresentam sintomas de sífilis, o que reforça a importância de se instituir o tratamento adequado ainda no início do pré-natal (Ensari et al., 2015).

Também pode ser observado ao longo dos anos, que as variáveis faixa etária, teste não-treponêmico e esquema de tratamento da gestante, apresentaram um nível de significância menor que 5\%, isto é, apresentaram uma tendência de aumento ao longo do período estudado. Espera-se que este fato se deva a melhoria da qualidade do serviço oferecido, principalmente na 
realização de exame não treponêmico (VDRL) na triagem do pré-natal, no ano 2015.

Para o diagnóstico da sífilis gestacional, a maioria das gestantes realizaram o teste não treponêmico (VDRL) como triagem no pré-natal. Estes achados demonstram que ainda persistem gestantes positivas com falha no diagnóstico no período do pré-natal, como preconizado pelo Ministério da Saúde. Nesse sentido, é importante que as pacientes entendam que a sífilis não deve ser uma preocupação apenas quando a mulher está grávida. O ideal é que, durante as consultas ginecológicas, a prevenção e o rastreamento desses casos se tornem uma rotina, despertando assim, uma reflexão e reestruturação da assistência pré-natal, e consequentemente reduzindo o percentual de diagnósticos e tratamentos não realizados (Simoura et al., 2010).

Os achados sobre o tratamento dos parceiros podem demonstrar uma possível falha na busca ativa desta população. No estado de Tocantins em estudo semelhante, 29,8\% dos parceiros não foram tratados, sendo este um dos fatores que favorecem a manutenção da doença no local estudado e ocorrência da sífilis congênita (Cavalcante et al., 2017).

A sífilis deve ser rastreada na primeira consulta de pré-natal para todas as gestantes, sendo repetidos os exames para rastreamento com 28 semanas de gestação e novamente antes da internação para realização do parto. Contrapondo os resultados encontrados no presente estudo, onde os mesmos dão suporte a hipótese de que o aumento da incidência deste agravo pode ser real, como reflexo de deficiências na assistência ao pré-natal oferecida as gestantes (Miranda et al., 2012).

Os resultados expuseram que houve tratamento realizado de forma inadequada ou ignorado, e parceiros que não foram tratados. Estudos mostraram algumas falhas que influenciam nessa realidade, dentre elas, a acessibilidade prejudicada aos serviços de saúde, falta de orientação, promoção e educação em saúde para a população, diagnóstico tardio da sífilis na mulher não gestante, ou na fase inicial da gestação e baixa adesão das mulheres grávidas ao serviço e assitência de pré-natal. Resultados similares foram relatados nos Estados Unidos e em Montes Claros, Minas Gerais. A triagem pré-natal e o tratamento adequado são os mais eficazes, impactando na redução da morbidade e taxas de mortalidade entre crianças (Gust et al., 2002; Lafetá et al., 2016; Wallace et al., 2015).

Neste estudo, a maioria dos parceiros não realizaram o tratamento, o que evidencia que possivelmente mesmo que a gestante/mãe seja tratada adequadamente pelas equipes de saúde da família, esta permanece exposta à recontaminação, pois o seu parceiro não é tratado, por desinteresse ou resistência ao tratamento, e/ou promiscuidade e futuramente desencadeia a sífilis congênita. Resultados semelhantes foram observados em dois estudos de Porto Alegre que $98 \%$ dos parceiros das gestantes positivas também não receberam nenhum tratamento, o que levou ao aumento dos casos tanto em gestante como em recémnascidos no período avaliado, esta elevação regular de notificações de sífilis congênita, estão relacionadas à transmissão sexual, seguida pela transmissão vertical no período gestacional (Lafetá et al., 2016; Lenz e Flores, 2011).

Além disso, é possível notar uma disparidade entre o número de gestantes notificadas com sífilis e mães de crianças com sífilis, o que demonstra a fragilidade do serviço de saúde do Município de Nossa Senhora do Socorro. Frente essa realidade faz-se necessário criar estratégias de sensibilização e responsabilização permanente dos profissionais de saúde, visando a detecção e tratamento adequado da sífilis durante a gravidez, interpretação correta dos testes e manejo adequado dos parceiros (Brasil, 2015; Milanez, 2016). A subnotificação também foi observada, na qual os profissionais desconheciam a sua necessidade (Lazarini e Barbosa, 2017).

Ao avaliar a associação entre as características sociodemográficas maternas e a ocorrência de sífilis congênita, podese observar que todas as variáveis puderam ser consideradas fatores de risco para a ocorrência da sífilis congênita, corroborando com diversos estudos, que destacam a baixa escolaridade como um fator de risco para sífilis, isto é, há uma relação inversa entre escolaridade e incidência de sífilis, demonstrando que estes fatores sociodemográficos podem evidenciar uma maior vulnerabilidade social, podendo inclusive denunciar o acesso precário a serviços de saúde, ou um limitado entendimento da importância das medidas de prevenção devido ao menor acesso à informação (Rodrigues et al., 2008).

Esta predominância de ocorrência da sífilis em população em vulnerabilidade social também foi descrita na Europa 
Ocidental e nos EUA (Fenton et al., 2008; Rodrigues et al., 2008; Domingues et al., 2013; Serafim et al., 2014; Cavalcante et al., 2017). Esta maior vulnerabilidade social também está intimamente relacionada à variável raça, em especial a negra e parda, uma vez que se trata de uma população historicamente desamparada (Santos, 2016). Desta forma, a sífilis congênita só será controlada efetivamente se houverem ações conjuntas a fim de reduzir as iniquidades sociais e melhorar a qualidade e a cobertura da assistência pré-natal (Figueiró et al., 2007; Domingues et al., 2013; Lima et al., 2013; Soeiro et al., 2014).

Quanto à variável estado civil, é interessante destacar que ao contrário de outros trabalhos que citam o estado civil solteira como fator de risco, alegando a instabilidade nos relacionamentos e a constante mudança das parcerias sexuais como fatores determinantes da infecção, este trabalho destaca ser casada como fator de risco (Silva et al., 2017). Ao que parece esta população não utiliza preservativo nas relações sexuais, por acreditarem que possuem relacionamento mais estáveis, e maior confiança nos parceiros (Macêdo et al., 2017).

\section{Conclusão}

A análise dos dados de sífilis na gestação e congênita no município de Nossa Senhora do Socorro - SE, possibilitou concluir que estes agravos persistem como problema de saúde pública, uma vez que as incidências anual e acumulada no período estudado foram ascendentes, estando associada à maior vulnerabilidade social e que a transmissão vertical esteve relacionada a perdas de oportunidades diagnósticas e terapêuticas.

Outro fator preocupante foi a evidência de possíveis problemas quanto ao diagnóstico precoce e tratamento adequado das gestantes e recém-nascidos. Apesar do pré-natal ter sido realizado por grande parte das mulheres, o diagnóstico durante esta etapa não se deu em mesma proporção, demonstrando falha no diagnóstico precoce dos casos de sífilis gestacional. Além disso, a maior parte das mulheres e seus companheiros não foram tratados corretamente.

Os altos índices epidemiológicos resultantes dessa pesquisa sugerem falhas na assistência pré-natal e indicam que ainda há um longo caminho a ser percorrido para que as taxas de sífilis congênita atinjam as preconizadas pela OPAS e Ministério da Saúde, portanto, esses agravos devem continuar sendo alvo de estudos a fim de gerarem novas estratégias de prevenção para reduzir a transmissão vertical da doença.

\section{Referências}

Brasil. Ministério da Saúde. (2005). Guia de Vigilância Epidemiológica. http://bvsms.saude.gov.br/bvs/publicacoes/Guia_Vig_Epid_novo2.pdf

Brasil. Ministério da Saúde. (2006). Programa Nacional de DST/AIDS. Diretrizes para controle da sífilis congênita: manual de bolso. https://bvsms.saude.gov.br/bvs/publicacoes/manual_sifilis_bolso.pdf

Brasil. Ministério da Saúde. (2015). Protocolo clínica e diretrizes terapêuticas para atenção integral às pessoas com infecção sexualmente transmissíveis. http://www.aids.gov.br/pt-br/pub/2015/protocolo-clinico-e-diretrizes-terapeuticas-para-atencao-integral-pessoas-com-infeccoes

Brasil. Ministério da Saúde. (2016). Boletim Epidemiológico. n n 35. http://www.aids.gov.br/system/tdf/pub/2016/59222/2016_030_sifili s_publicao2_pdf_51905.pdf?file=1\&type=node $\&$ id $=59222 \&$ force $=1$

Campos, A. L. A., Araújo, M. A. L., Melo, S. P. \& Gonçalves, M. L. C. (2010). Epidemiologia da sífilis gestacional em Fortaleza, Ceará, Brasil: um agravo sem controle. Cadernos de Saúde Pública, 26 (9), 1747-1755.

Cavalcante, P. A. M., Pereira, R. B. L., \& Castro, J. G. D. (2017). Sífilis gestacional e congênita em Palmas, Tocantins, 2007-2014. Epidemiologia e Serviços de Saúde, 26 (2), 255-264.

Domingues, R. M. S. M., Saraceni, V., Hartz, Z. M. A. \& Leal, M. C. (2013). Sífilis congênita: evento sentinela da quantidade de assistência pré-natal. Revista de Saúde Pública, 47 (7), 147-157.

Ensari, T., Kirbas, A., Ozgu-Erdinc, A. S., Saygan, S. G., Erkaya, S., Uygur, D., et al. (2015). An eight-year retrospective analysis of antenatal screening results for syphilis: is it still cost effective? The Journal of Infection in Developing Countries, 9 (9), 1011-1015.

Fenton, K. A., Breban, R., Vardavas, R., Okano, J. T., Martin, T., Aral, S., et al. (2008). Infectious syphilis in high-income settings in the 21st century. The Lancet Infectious Diseases. 8, 244-253. 
Figueiró, E. A., Filho, Gardenal, R. V. C., Assunção, L. A., Costa, G. R., Periotto, C. R. L., Vedovatte, C. A., et al. (2007). Sífilis congênita como fator de assistência pré-natal no município de Campo Grande - MS. DST Jornal Brasileiro de Doenças Sexualmente Transmissíveis, 19 (3-4), $139-143$.

Gust, D. A., Levine, W. C., Louis, M. E. S., Braxton, J. \& Berman, S. M. (2002). Mortality Associated With Congenital Syphilis in the United States, 19921998. Pediatrics, 109 (5), 79-86.

Lafetá, K. R. G., Martelli, H., Júnior, Silveira, M. F. \& Paranaíba, L. M. R. (2016). Sífilis materna e congênita, subnotificação e difícil controle. Revista Brasileira de Epidemiologia, 19 (1), 63-74.

Lazarini, F. M. \& Barbosa, D. A. (2017). Educational intervention in Primary Care for the prevention of congenital syphilis. Revista Latino-Americana de Enfermagem, 25, e2845.

Leitão, E. J. L., Canedo, M. C. M., Furiatti, M. F., Oliveira, L. R. S., Diener, L. S., Lobo, M. P., et al. (2009). Sífilis gestacional como indicador da qualidade do pré-natal no Centro de Saúde de Samambaia - DF. Revista Comunicação em Ciências da Saúde, 4 (20), $306-314$.

Lenz, M. L. M. \& Flores, R. (2011). Atenção à saúde da gestante em Atenção Primária à Saúde. Porto Alegre: Hospital Nossa Senhora da Conceição.

Lima, M. G., Santos, R. F. R., Barbosa, G. J. A., Ribeiro, G. S. (2013). Incidência e fatores de risco para sífilis congênita em Belo Horizonte, Minas Gerais, 2001-2008. Revista Ciência \& Saúde Coletiva, 18 (2), 499-506.

Macêdo, V. C., Lira, P. I. C., Frias, P. G., Romaguera, L. M. D., Caires, S. F. F. \& Ximenes, R. A. A. (2017). Fatores de ris co para sífilis em mulheres: estudo caso-controle. Revista de Saúde Pública, 51, 1-12.

Mesquita, K. O., Lima, G. K., Filgueira, A. A., Flôr, S. M. C., Freitas, C. A. S. L., Linhares, M. S. C., et al. (2012). Análise dos casos de sífilis congênita em sobral, Ceará: contribuições para assistência pré-natal. DST Jornal Brasileiro de Doenças Sexualmente Transmissíveis, 24 (1), $20-27$.

Milanez, H. (2016). Syphilis in Pregnancy and Congenital Syphilis: Why Can We not yet Face This Problem? Revista Brasileira de Ginecologia e Obstetrícia, 38 (9), 425-427.

Miranda, M. M. S., Souza, L. M. G., Aguiar, R. A. L. P., Corrêa, M. D. J., Maia, M. M. M., Borges, R. S., et al. (2012). Rastreamento das infecções perinatais na gravidez: realizar ou não? Femina, 40 (1), 13-22.

Mullick, S., Beksinksa, M. \& Msomi, S. (2005). Treatment for syphilis in antenatal care: compliance with the three dose standard treatment regimen. Sexually Transmitted Infections, 81, 220-222.

Newman, L., Kamb, M., Hawkes, S., Gomez, G., Say, L., Seuc, A., et al. (2013). Global estimates of syphilis in pregnancy and associated adverse outcomes: analysis of multinational antenatal surveillance data. PLOS Medicine, 10 (2), e1001396.

Organização Pan Americana de Saúde. (2010). Análisis de la situación al año 2010: eliminación de la transmisión materno infantil del VIH y de la sífilis congénita em la Región de las Américas. https://iris.paho.org/handle/10665.2/49413

Organização Pan Americana de Saúde. (2014). Field guide for implementation of the strategy and plan of action for elimination of mother-to-child transmission of HIV and congenital syphilis in the Americas. https://iris.paho.org/handle/10665.2/6080?locale-attribute=pt

Rodrigues, C. S., Guimarães, M. D. C. \& Cesar, C. C. (2008). Oportunidades perdidas de prevenção da sífilis congênita e HIV: transmissão perinatal. Revista de Saúde Pública, 42 (5), 851-858.

Santos, N. J. S. (2016). Mulher e negra: dupla vulnerabilidade as DST/HIV/Aids. Saúde e Sociedade, 25 (3), $602-618$.

Serafim, A. S., Moretti, G. P., Serafim, G. S., Niero, C. V., Rosa, M. I., Pires, M. M. S., et al. (2014). Incidence of congenital syphilis in the South Region of Brazil. Revista da Sociedade Brasileira de Medicina Tropical, 47 (2), 170-178.

Sheffield, J. S., Sánchez, P. J., Morris, G., Maberry, M., Zeray, F., Mcintire, D. D., et al. (2002). Congenital syphilis after maternal treatment for syphilis during pregnancy. Amerincan Journal of Obstetrics and Gynecology, 186 (3), 568-573.

Silva, M. R. F., Brito, E. S. V., Freire, L. C. G., Pedrosa, M. M., Sales, V. M. B. \& Lages, I. (2010). Percepção de mulheres com relação a ocorrência da sífilis congênita em seus conceptos. Revista Ação Social do Paraná, 13 (3), 301-309.

Silva, Z. F., Teixeira, K. S. S. \& Nascimento, D. S. (2017). Pacientes portadores de sífilis atendidos em uma unidade terciária em Fortaleza: perfil sociodemográfico. Revista Brasileira de Análises Clínicas, 49 (1), 105-109.

Simoura, A. A., Coelho, M. M., Coelho, S. M., Hernandez, D. P. \& Albuquerque, V. S. (2010). A enfermagem e o saber das gestantes em assistência pré-natal acerca da sífilis e sífilis congênita. http://www.repositorio.ufc.br/handle/riufc/21661

Soeiro, C. M. O., Miranda, A. E., Saraceni, V., Santos, M. C., Talhari, S. \& Ferreira, L. C. L. (2014). Syphilis in pregnancy and congenital syphilis in Amazonas State, Brazil: an evaluation using database linkage. Cadernos de Saúde Pública, 30 (4), 715-723.

Wallace, H. E., Broomhall, H. M., Isitt, C. I., Miall, L. S. \& Wilson, J. D. (2015). Serological follow-up of infants born to mothers with positive syphilis serology-real-world experiences.International Journal of STD \& AIDS, 15, 1-5.

World Health Organization. (2014). Global guidence on criteria and processes for validation: elimination of mother-to-child tranmission (EMTCT) of HIV and syphilis. https://www.who.int/reproductivehealth/publications/emtct-hiv-syphilis/en/

World Health Organization. (2016). Sexually transmitted infections (STIs). http://www.who.int/mediacentre/factsheets/fs110/en/ 